\title{
Evaluación de nódulos tiroideos con interpretación citológica rápida en el sitio (ROSE)
}

\section{Evaluation of thyroid nodules with Rapid On-Site Evaluation (ROSE)}

\author{
Laura Joanna Tapia-Vela1', Lina Restrepo-Giraldo²
}

Resumen. La aspiración con aguja fina (ACAF) es una técnica ampliamente utilizada por su alta seguridad y fácil uso. Sin embargo, al usarse en conjunto con la evaluación rápida de la muestra en el sitio de la toma (ROSE, del inglés, Rapid OnSite Evaluation), disminuyen los tiempos necesarios para la evaluación y el diagnóstico, mejora la calidad de la muestra, disminuye el número de punciones por procedimiento y limita la necesidad de repetir la prueba, lo que hace que el uso combinado de ambas técnicas sea de gran utilidad y cada vez más solicitado en los servicios de imágenes diagnósticas y patología. ROSE, además, permite una clasificación adecuada del material recolectado para cultivos, estudios de citometría de flujo y pruebas moleculares. Particularmente, en los pacientes con nódulos tiroideos, la realización de ROSE durante el procedimiento de evaluación ecográfica y punción, puede garantizar que la muestra extraída sea suficiente y adecuada para el diagnóstico, y, así mismo, permite evaluar la necesidad de estudios complementarios de manera inmediata, brindando un diagnóstico rápido y preciso, disminuyendo los costos relacionados con la repetición del procedimiento y la morbilidad asociada a complicaciones por punciones múltiples.

Palabras clave: biopsia por aspiración con aguja fina, nódulo tiroideo, muestra, evaluación, patología, diagnóstico.

Abstract. Fine needle aspiration (FNA) is a widely used technique due to its high safety and ease of use. However, when used in combination with Rapid On-Site Evaluation (ROSE), there is a reduction in the time required for evaluation and diagnosis, as well as an improvement in the sample quality, a reduction in the number of needle passes and the need to repeat the test, which makes the combined

\footnotetext{
${ }^{1}$ Médica, Especialista en Patología. Jefe de Patología Anatómica, Laboratorio Clínico Hematológico. Medellín, Colombia. E-mail: Itapia@hematologico.com.

${ }^{2}$ Médica, Especialista en Medicina Interna, Especialista en Endocrinología Clínica y Metabolismo, MSc en Oncología. Jefe de Enlace Clínico, Laboratorio Clínico Hematológico. Medellín, Colombia. 
use of both techniques very useful and increasingly requested in the diagnostic imaging and pathology services. ROSE also allows a proper classification of the collected material for cultures, flow cytometry studies and molecular tests. Particularly in patients with thyroid nodules, the performance of ROSE during the ultrasound evaluation and biopsy procedure can guarantee that the sample extracted is sufficient and adequate for the diagnosis, and, likewise, an immediate evaluation for the need for complementary studies can be achieved, providing a fast and accurate diagnosis, reducing the costs related to repeating the procedure, and the morbidity associated with complications from multiple biopsies.

Keywords: fine-needle biopsy, thyroid nodule, sample, evaluation, pathology, diagnosis.

\section{Introducción}

La aspiración con aguja fina (ACAF) para la extracción de parte o totalidad de tejidos, se considera un procedimiento sencillo de realizar, altamente seguro y capaz de brindar una muy valiosa y relevante información diagnóstica [1], lo que la hace el método de elección para el estudio de nódulos en diversas localizaciones anatómicas. El acompañamiento con imágenes diagnósticas, principalmente ecografía, aumenta indiscutiblemente su rendimiento y precisión [2-4]. No obstante, una de las limitaciones es la amplia variabilidad en las tasas de extracción del material adecuado y suficiente para el estudio citopatológico, muy dependiente de la técnica usada y la experticia del operador $[5,6]$.

El uso de la evaluación citológica rápida en el sitio (ROSE, del inglés, Rapid On-Site Evaluation) es cada vez más extendido. Es una estrategia de los servicios de citología, usada principalmente durante procedimientos mínimamente invasivos, como la ACAF, para evaluar la suficiencia de la muestra tomada [7], haciendo que el procedimiento sea mucho más eficiente y con mayor rentabilidad diagnóstica $[8,9]$. Se espera que un procedimiento de biopsia produzca no solo material para el diagnóstico, sino que sea suficiente para realizar pruebas complementarias y estudios moleculares, con objetivos pronósticos y terapéuticos. El uso de ROSE, además, permite la comunicación directa entre el patólogo y el médico durante el procedimiento de extracción de la muestra, y mejora la probabilidad de un diagnóstico preciso, completo y oportuno [10]. Por último, cabe resaltar que el hecho de que una muestra sea adecuada para evaluación, no significa que es suficiente para el diagnóstico, ya que esto depende también del rendimiento y la precisión de la citología, relacionados, a su vez, con la interpretación y el diagnóstico [11].

Entre las principales ventajas de realizar la ACAF y ROSE en conjunto, se encuentran la posibilidad de disminuir los tiempos necesarios para la evaluación y el diagnóstico, mejorar la calidad de la muestra, disminuir el número de punciones por procedimiento y limitar la necesidad de repetir la prueba, lo que hace que su uso combinado sea de gran utilidad y cada vez más solicitado en los servicios de imágenes diag- 
nósticas y patología. ROSE permite una clasificación adecuada del material recolectado, lo que indica que se dispone del material suficiente para cultivos, estudios de citometría de flujo y pruebas moleculares [12,13].

La presencia del patólogo durante ROSE lo acerca al paciente y a otros médicos. El estar juntos en un procedimiento, brinda la oportunidad de compartir información sobre la historia clínica, revisar las imágenes radiológicas y discutir los resultados de la aspiración, lo que mejora el buen cuidado del paciente.

\section{Ecografía y ACAF de nódulos tiroideos}

Uno de los sitios que se punciona con mayor frecuencia es la glándula tiroides, donde la ACAF se considera la prueba de elección para el estudio de los nódulos presentes en ella. Los nódulos tiroideos son un problema clínico común; son tan frecuentes que se pueden detectar en el 19\% al 68\% de los individuos de la población seleccionados al azar, con mayor frecuencia en mujeres y ancianos. La ecografía es el estudio imagenológico de elección para detectar y caracterizar los nódulos tiroideos [16], ya que proporciona información sobre el tamaño del nódulo, la ubicación, las características ecográficas, incluidas la composición, ecogenicidad, márgenes, presencia y tipo de calcificaciones, forma (si es más alta que ancha) y vascularización [17]. Además, se puede utilizar para describir la presencia de adenopatías [18]. La combinación de las características ecográficas y el tamaño del nódulo, puede orientar a los médicos sobre las indicaciones para realizar la ACAF para el diagnóstico citológico $[6,8,19,20]$.

\section{Clasificación de los nódulos tiroideos}

Existen sistemas de clasificación de los nódulos tiroideos que permiten definir cuáles son candidatos para ACAF de tiroides, por ejemplo, los propuestos en las guías de la Asociación Americana de Tiroides (ATA, por sus siglas en inglés) $[21,22]$ y la clasificación TI-RADS (del inglés, Thyroid Imaging-Reporting and Data System) 2017 del Colegio Americano de Radiología (ACR, por sus siglas en inglés) [21,23]; ambos basados en las características encontradas en la ecografía. En el protocolo inicial de estudio del nódulo tiroideo se recomienda evaluar la TSH, ya que si está baja o suprimida se debe descartar un nódulo hiperfuncionante antes de realizar ACAF, debido a que estos nódulos autónomos (adenomas tóxicos) casi siempre son benignos, pero sus características como la hiperplasia pueden generar confusión en el citodiagnóstico [24,25]. Dado el bajo riesgo de malignidad y los potenciales falsos positivos, no se recomienda realizar ACAF en adenomas tóxicos. También se desaconseja la aspiración de nódulos menores a $1 \mathrm{~cm}$, a menos que el paciente tenga un historial de alto riesgo, como antecedente personal de cáncer de tiroides en el lóbulo contralateral, antecedentes familiares de primer grado de cáncer de tiroides, historia de irradiación o sospecha de carcinoma medular de tiroides [26].

La ATA estratifica los nódulos tiroideos en cinco categorías de riesgo de malignidad: alta sospecha (riesgo estimado de $70 \%$ a $90 \%$ ), sospecha intermedia (10\% a $20 \%)$, baja sospecha (5\% a $10 \%)$, muy baja sospecha $(<3 \%)$ y benigno (quiste) (tabla 1). Además, recomienda realizar la ACAF diagnóstica para nódulos tiroideos de sospecha alta o intermedia que sean de 1 
Tabla 1. Clasificación de los nódulos tiroideos de acuerdo con ATA 2015 y TI-RADS $2017[21,27]$

\begin{tabular}{l} 
Clasificación ATA 2015 \\
\hline Alta sospecha (riesgo de malignidad 70\% \\
a 90\%) \\
Nódulo sólido hipoecoico con una o más de \\
las siguientes características: \\
- Márgenes irregulares \\
- Microcalcificaciones \\
- Más alto que ancho \\
- Borde calcificado con extrusión de \\
tejidos blandos \\
- Extensión extratiroidea
\end{tabular}

Sospecha intermedia (riesgo de malignidad $10 \%$ a $20 \%$ )

Nódulo hipoecoico con márgenes definidas

Baja sospecha (riesgo de malignidad $5 \%$ a $10 \%$ )

Nódulo isoecoico o hiperecoico sólido o parcialmente quístico con áreas sólidas excéntricas

Muy baja sospecha (riesgo de malignidad $<3 \%$ )

Nódulo parcialmente quístico y espongiforme

\section{Benigno}

Completamente quístico

\section{ACR TI-RADS 2017}

TR5 (alta sospecha, $>20 \%$ riesgo de malignidad)

Puntaje TI-RADS $\geq 7$ puntos

TR4 (sospecha moderada, $5 \%$ a $20 \%$ de riesgo de malignidad)

Puntaje TI-RADS 4 a 6 puntos

TR3 (leve sospecha, $5 \%$ de riesgo de malignidad)

Puntaje TI-RADS 3 puntos

TR2 (no sospechoso, $<2 \%$ riesgo de malignidad)

Puntaje TI-RADS 2 puntos

TR1 (benigno, $\leq 2 \%$ riesgo de malignidad)

Puntaje TI-RADS 0 puntos cm o más, de baja sospecha de 1,5 cm o más, y de muy baja sospecha de 2 $\mathrm{cm}$ o más [7].

En 2009 se introdujo el sistema de clasificación ecográfico TI-RADS para los hallazgos encontrados en los nódulos tiroideos. En 2017, la Asociación Europea de Tiroides propuso el EU-TI-RADS y el ACR presentó el ACR TI-RADS $[26,28]$. En el sistema de puntuación ACR TI-RADS se otorgan puntos por diferentes características ecográficas, incluyendo la composición del nódulo, la ecogenicidad, la forma, el margen y la presencia de focos ecogénicos (figura 1). El número total de puntos es una indicación del nivel de riesgo TI-RADS; así, hay cinco niveles ACR TI-RADS, cada uno de los cuales tiene un diferente riesgo asociado de malignidad (tabla 1) [29]. El sistema ACR TI-RADS recomienda la ACAF para nódulos tiroideos con puntaje TR3 con tamaño mayor o igual a 2,5 $\mathrm{cm}$, TR4 de tamaño mayor o igual a 1,5 $\mathrm{cm}$, y TR5 con tamaño mayor o igual a $1 \mathrm{~cm}$. No se recomienda realizar ACAF diagnóstica para los nódulos TR1 y TR2 (figura 1) [23,28,30].

La principal ventaja de la ACAF de tiroides radica en su capacidad para identificar nódulos benignos y malignos con un elevado grado de certeza. Una citología benigna en ACAF tiene un valor predictivo negativo que supera el $95 \%$, y la citología maligna tiene un valor predictivo positivo que excede el $99 \%$ [31]. Dirigir la punción por ecografía puede disminuir los falsos negativos al $1 \%$ o $2 \%$ de las muestras [32]. 


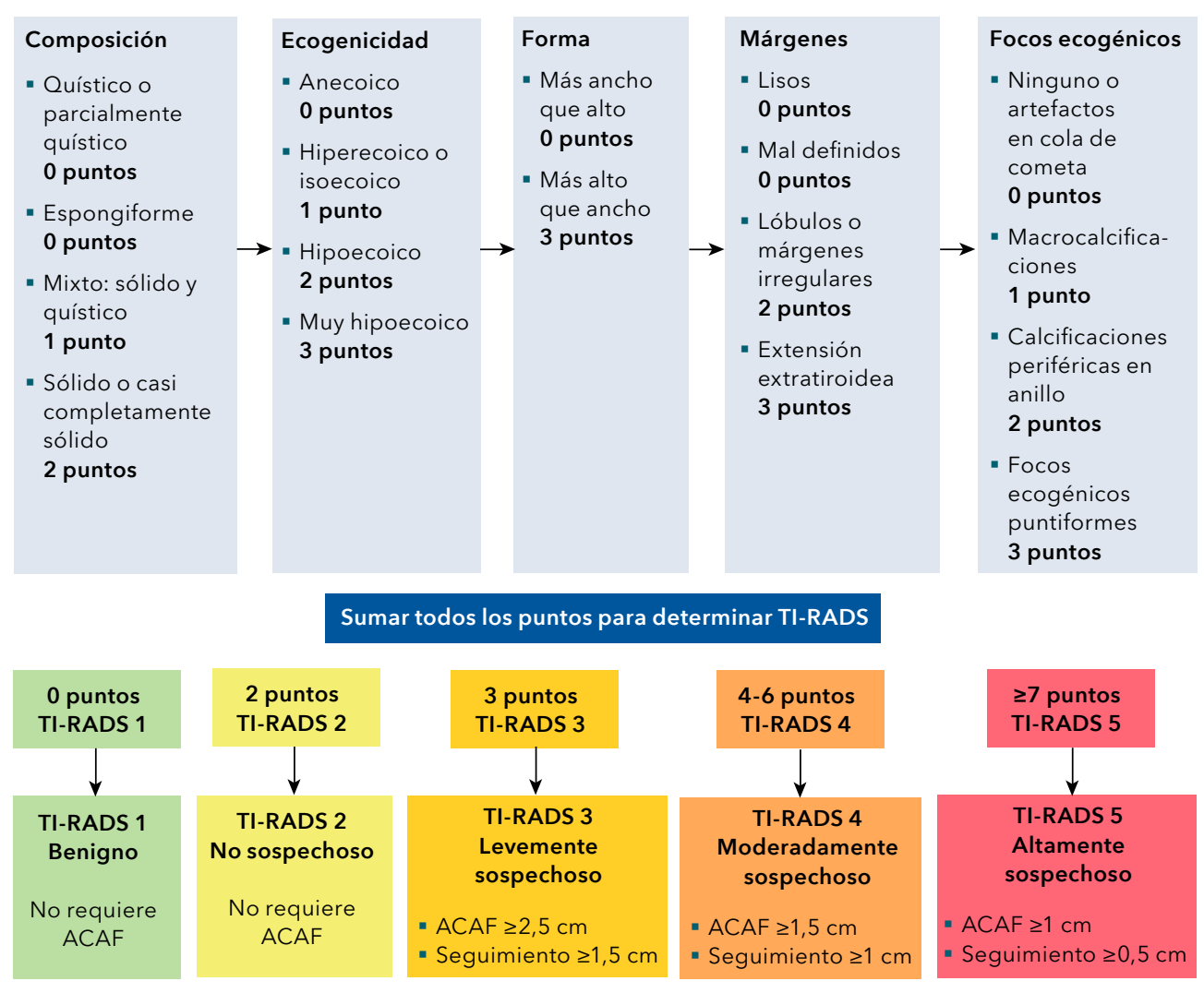

Figura 1. Sistema de puntuación ACR TI-RADS 2017 para los nódulos tiroideos y conducta a seguir según el riesgo dado por el cálculo ecográfico [23].

Obtener material adecuado a veces puede ser muy difícil debido a lo vascularizado que se encuentra el tejido y a la frecuente presencia de degeneración quística de los nódulos, que puede llevar a que la muestra sea no diagnóstica en el $25 \%$ al $50 \%$ de los casos. Incluso con personal altamente entrenado, se reporta que hasta un $15 \%$ de los aspirados tiroideos son no diagnósticos [32].

La asistencia al procedimiento por patología se utiliza en muchos centros como medio para mejorar las tasas de precisión y adecuación, asegurando una preparación más óptima de la muestra. ROSE permite la evaluación de la representatividad de la muestra y determina la necesidad de recolectar material adicional para estudios complementarios durante la misma atención del paciente (figura 2) [20,26]. Finalmente, definir la conducta terapéutica de acuerdo al resultado del estudio citológico del nódulo, ha disminuido el número de tiroidectomías innecesarias en un $50 \%[6,32]$.

\section{Técnica en tiroides}

La ACAF implica la inserción de una aguja de calibre 22 en el nódulo tiroideo sospechoso, seguido de un movimiento oscilante a través del nódulo. 


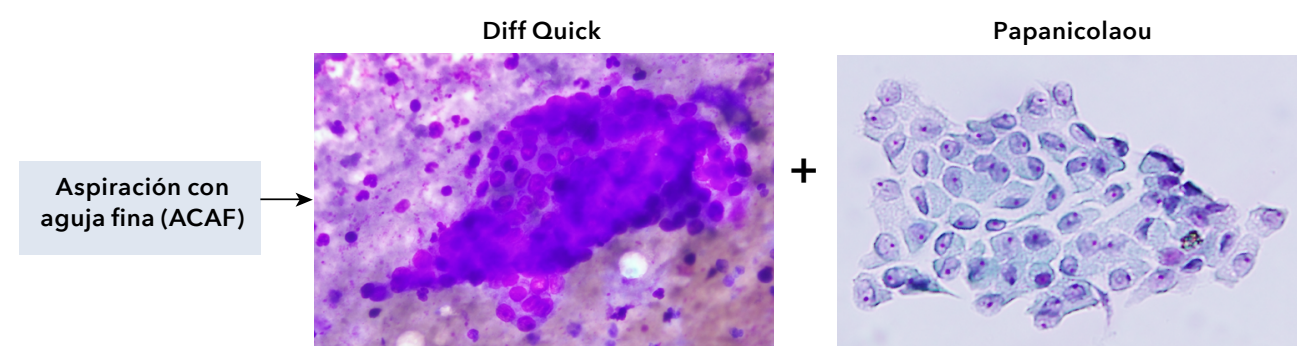

ROSE (nódulo tiroideo)

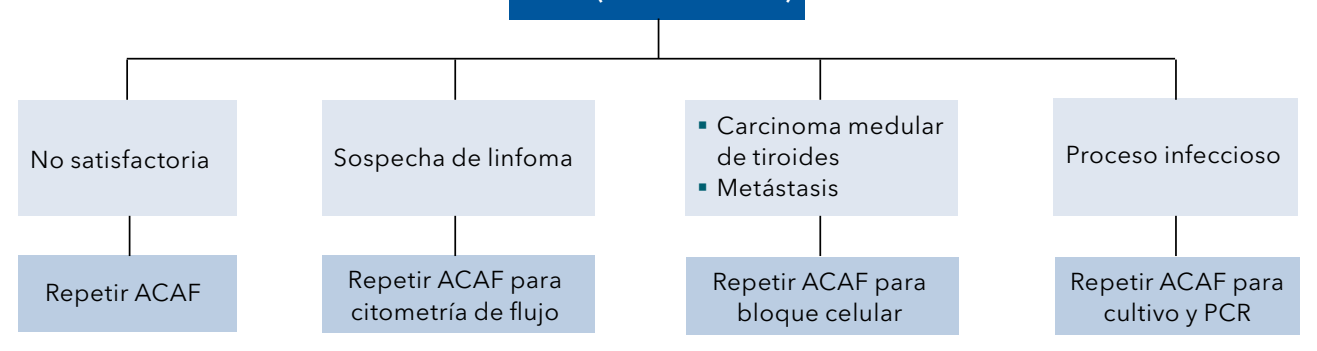

Figura 2. Optimización de la muestra para realizar estudios complementarios según la aproximación diagnóstica. Láminas de citología de tiroides coloreadas con técnicas de Diff Quick y Papanicolaou. Tomado y modificado [31].

El acompañamiento del procedimiento con ecografía aumenta claramente el rendimiento diagnóstico en comparación con el guiado únicamente por palpación, mejora la seguridad de la biopsia de nódulos no palpables, y brinda pistas diagnósticas útiles sobre la biología y el tamaño del nódulo [20]. Hallazgos en el nódulo como microcalcificaciones, hipoecogenicidad, márgenes irregulares, flujo intranodular y una apariencia más alta que ancha son características que se asocian con malignidad. Aunque no tienen un valor predictivo de forma independiente, estos hallazgos pueden orientar la decisión de realizar una biopsia o dirigir la biopsia a los nódulos más sospechosos en casos de multinodularidad [22,26].

Aunque el manejo del material obtenido es sencillo, se debe hacer de manera rápida y correcta. Los retrasos y errores en la manipulación de la mues- tra después del retiro de la aguja de la tiroides, pueden causar artefactos en la preparación que alteran el proceso diagnóstico y disminuyen las tasas de extracción de material suficiente [33]. Se recomienda que el material aspirado sea expulsado de la aguja fina dentro de los 30 segundos posteriores a la finalización de la aspiración, para evitar la coagulación de la sangre, ya que la sangre coagulada atrapa las células y limita la evaluación morfológica y otros estudios adicionales [26].

Cuando se realiza correctamente, la evaluación por ROSE mejora la calidad de las muestras, disminuye la necesidad de repetir el procedimiento y acorta el tiempo que podría tardar el proceso diagnóstico convencional, ya que cuando estas pruebas no se realizan en conjunto, el paciente debe regresar al centro médico en múltiples oportunidades, corriéndose el riesgo de que el procedimiento no sea exito- 
so y se deba esperar hasta tres meses para repetirlo y obtener el diagnóstico, lo que prolonga el inicio del tratamiento $[3,26]$.

\section{Otras aplicaciones}

\section{Glándula salival}

El examen clínico de los nódulos en las glándulas salivales mayores, como la parótida, no es suficiente para distinguir los quistes de los tumores, por lo cual, a menudo se requiere que sea acompañado de procedimientos de imagen. Las técnicas de imágenes pueden proporcionar información precisa sobre la naturaleza, el tamaño, el lugar de los nódulos y la apariencia de la glándula. Todo lo anterior hace que el estudio de citología obtenida por ACAF guiada por imágenes, conduzca a diagnósticos preoperatorios precisos en las glándulas salivales, además de ser seguro, fácil de realizar y causar pocas molestias al paciente [34,35].

\section{Pulmón}

El procedimiento de aspiración se realiza utilizando un broncoscopio para obtener muestras de tejido o líquido de los pulmones, o de los ganglios linfáticos cercanos. La aspiración se puede realizar bajo guía tomográfica o ecográfica (ecografía endobronquial o EBUS) [36]. La EBUS es un procedimiento muy eficaz que se utiliza para diagnosticar el cáncer de pulmón, las infecciones y otras enfermedades que provocan el crecimiento de los ganglios en el tórax [37]. Para los tumores inoperables, a veces la citología es la única herramienta válida tanto para el diagnóstico como para la caracterización biomolecular. ROSE es una técnica útil para evaluar rápidamente la idoneidad del material biológico obtenido, en términos de calidad y cantidad para el diagnóstico, y para proporcionar información biológica que pueda utilizarse para definir la terapia [36,38,39].

\section{Hígado}

En las lesiones viscerales, la ACAF se puede realizar con guía ecográfica, ecografía endoscópica o tomográfica computarizada, como alternativa a otros métodos diagnósticos más invasivos $[40,41]$. Específicamente, la ACAF de hígado bajo guía radiológica ha sido considerada un procedimiento complejo, con factores que pueden interferir con su resultado, como la naturaleza y ubicación de la lesión, y la experiencia y habilidad del operador [42]. ROSE ha mostrado aumentar hasta un $90 \%$ el rendimiento diagnóstico de las ACAF radiológicamente guiadas, y reducir la necesidad de repetir el procedimiento hasta en un 30\%. Así mismo, permite decidir el número de punciones realizadas durante el mismo procedimiento y reducir el costo de la atención médica, al evitar la necesidad de una biopsia abierta, lo que aumenta la confianza de los médicos en la ACAF como un procedimiento de diagnóstico mínimamente invasivo y bien tolerado por los pacientes [43].

\section{Páncreas}

La ACAF guiada por ecoendoscopia y acompañada por ROSE, puede proporcionar un diagnóstico rápido y preciso [44]. Este diagnóstico rápido ayuda a ganar tiempo adicional para asesorar al paciente e iniciar un estudio preoperatorio, antes de que estén disponibles los resultados finales de la biopsia $[45,46]$. La técnica de ROSE es 
muy útil en la diferenciación de neoplasias, de hecho, su precisión diagnóstica para tumores benignos se estima que es cercana al $97 \%$, mientras que para las lesiones malignas es del 89,9\% [47].

\section{Telecitología y ROSE}

A medida que aumenta la cantidad de procedimientos mínimamente invasivos, también aumenta la cantidad de sitios que requieren ROSE, y, a su vez, un número mayor de personal con conocimiento de citología en las instituciones. La telecitología representa una solución razonable para aumentar la eficiencia del personal y permite al patólogo permanecer en el laboratorio, mientras un citotecnólogo envía las imágenes desde el lugar donde se está realizando el procedimiento [48]. La aparición de tecnologías de imágenes digitales rápidas y de alta resolución, ha hecho posible la telecitología y ha permitido hacer más eficiente el proceso [49]. En resumen, la telecitología para ROSE es actualmente adecuada para uso clínico en entornos de gran volumen, lo cual aumenta la eficiencia del tiempo sin detrimento en la calidad o atención al paciente [50,51].

\section{Estudios complementarios}

La evaluación citológica rápida en el momento de la ACAF optimiza la clasificación de la muestra para el diagnóstico y los estudios adicionales [10]. Con una planificación previa, la muestra se puede dividir para aumentar su rendimiento. La evaluación inmediata brinda la oportunidad de solicitar que se aumente la cantidad de muestra y clasificarla [39]. Aunque el examen microscópico sigue siendo el pilar del diagnóstico en citología, los patólogos ya están acostumbrados a complemen- tar la revisión morfológica con pruebas adicionales, por ejemplo, usando inmunohistoquímica, citogenética, hibridación in situ, citometría de flujo y pruebas moleculares [52,53].

\section{Conclusión}

La realización de ROSE en procedimientos mínimamente invasivos como la ACAF guiada por imágenes, ha mostrado ser de gran utilidad y de enorme potencial en el diagnóstico de nódulos en diversas localizaciones anatómicas. Particularmente en los pacientes con nódulos tiroideos, en quienes por las características clínicas y ecográficas se determina la necesidad de realizar estudios de ecografía y ACAF guiada por ecografía, la realización de ROSE durante el procedimiento de evaluación ecográfica y punción, puede garantizar que la muestra extraída sea suficiente y adecuada para el diagnóstico, así mismo, permite evaluar la necesidad de estudios complementarios de manera inmediata, brindando un diagnóstico rápido y preciso. Todo lo anterior permite realizar una aproximación diagnóstica y terapéutica oportuna, optimizando al máximo el tiempo necesario para la realización de estas pruebas.

\section{Referencias}

1. Díaz-Del Arco C, Ortega-Medina L, Fernández-Aceñero MJ. The role of fine-needle aspiration cytology in the diagnosis of soft tissue nodules: Experience in a tertiary center. Rev Esp Patol 2019;52:147-153. https://doi. org/10.1016/j.patol.2018.10.005.

2. Schmidt RL, Walker BS, Cohen MB. When is rapid on-site evaluation cost-effective for fine-needle aspiration biopsy? PLoS One 2015;10:e0135466. https://doi.org/10.1371/journal.pone.0135466.

3. Witt BL, Schmidt RL. Rapid onsite evaluation improves the adequacy of fine-needle aspi- 
ration for thyroid lesions: a systematic review and meta-analysis. Thyroid 2013;23:428-435. https://doi.org/10.1089/thy.2012.0211.

4. Ng DL, Vuhahula E, Zhang L, Waterhouse EG, White KL, Mushi BP, et al. Efficacy of an intensive, ultrasound-guided fine-needle aspiration biopsy training workshop in Tanzania. J Glob Oncol 2018;4:1-9. https://doi.org/10.1200/ jgo.18.00134.

5. Fetzer R, Duey M, Pena V, Wanzer D, Kirkpatrick J, Chau D, et al. Role of cytotechnologists in rapid onsite adequacy assessment of cytology materials for diagnostic workup and specimen allocation for ancillary testing using a standardized protocol. J Am Soc Cytopathol 2020;9:67-75. https://doi.org/10.1016/j. jasc.2019.08.005.

6. Zargham R, Johnson H, Anderson S, Ciolino A. Conditions associated with the need for additional needle passes in ultrasound-guided thyroid fine-needle aspiration with rapid on-site pathology evaluation. Diagn Cytopathol 2021;49:105108. https://doi.org/10.1002/dc.24605.

7. VanderLaan PA, Chen Y, Alex D, Balassanian R, Cuda J, Hoda RS, et al. Results from the 2019 American Society of Cytopathology survey on rapid on-site evaluation-part 1: objective practice patterns. J Am Soc Cytopathol 2019;8:333-341. https://doi.org/10.1016/j. jasc.2019.07.007.

8. Shield PW, Cosier J, Ellerby G, Gartrell M, Papadimos D. Rapid on-site evaluation of fine needle aspiration specimens by cytology scientists: a review of 3032 specimens. Cytopathology 2014;25:322-329. https://doi.org/10.1111/ cyt.12157.

9. Kothari K, Tummidi S, Agnihotri M, Sathe $P$, Naik L. This 'Rose' has no thorns-diagnostic utility of 'rapid on-site evaluation' (ROSE) in fine needle aspiration cytology. Indian J Surg Oncol 2019;10:688-698. https://doi.org/10.1007/ s13193-019-00981-y.

10. Stevenson T, Powari $\mathbf{M}$, Bowles $\mathbf{C}$. Evolution of a rapid onsite evaluation (ROSE) service for endobronchial ultrasound guided (EBUS) fine needle aspiration (FNA) cytology in a UK hospital: A 7 year audit. Diagn Cytopathol 2018;46:656-662. https://doi.org/10.1002/ dc. 23967 .
11. Sauter JL, Chen Y, Alex D, Balassanian R, Cuda J, Flanagan MB, et al. Results from the 2019 American Society of Cytopathology survey on rapid onsite evaluation (ROSE)-part 2: subjective views among the cytopathology community. J Am Soc Cytopathol 2020;9:570-578. https:// doi.org/10.1016/j.jasc.2020.06.010.

12. Schmidt RL, Witt BL, Lopez-Calderon LE, Layfield LJ. The influence of rapid onsite evaluation on the adequacy rate of fine-needle aspiration cytology: a systematic review and meta-analysis. Am J Clin Pathol 2013;139:300-308. https://doi.org/10.1309/ajcpegzmjkc42vup.

13. De Luca $C$, Sgariglia $R$, Nacchio $M$, Pisapia $P$, Migliatico I, Clery E, et al. Rapid on-site molecular evaluation in thyroid cytopathology: A same-day cytological and molecular diagnosis. Diagn Cytopathol 2020;48:300-307. https:// doi.org/10.1002/dc.24378.

14. Burman KD, Wartofsky L. Clinical practice. Thyroid Nodules. N Engl J Med 2015;373:2347-2356. https://doi.org/10.1056/ NEJMcp1415786.

15. Medina-Chamorro FM, Calle JA, Stein JE, Merchancano L, Mendoza-Briñez AM, PulidoWilches AA. Experience of the implementation of rapid on-site evaluation in ultrasound-guided fine-needle aspiration biopsy of thyroid nodules. Curr Probl Diagn Radiol 2018;47:220-224. https://doi.org/10.1067/j.cpradiol.2017.06.009.

16. Maxwell C, Sipos JA. Clinical diagnostic evaluation of thyroid nodules. Endocrinol Metab Clin North Am 2019;48:61-84. https://doi. org/10.1016/j.ecl.2018.11.001.

17. Grani G, Sponziello M, Pecce V, Ramundo V, Durante C. Contemporary thyroid nodule evaluation and management. J Clin Endocrinol Metab 2020;105:2869-2883. https://doi. org/10.1210/clinem/dgaa322.

18. Schlumberger M, Leboulleux S. Current practice in patients with differentiated thyroid cancer. Nat Rev Endocrinol 2021;17:176-188. https:// doi.org/10.1038/s41574-020-00448-z.

19. Vargas-Uricoechea H, Meza-Cabrera I, Herrera-Chaparro J. Concordance between the TIRADS ultrasound criteria and the BETHESDA cytology criteria on the nontoxic thyroid nodule. Thyroid Res 2017;10:1. https://doi.org/10.1186/ s13044-017-0037-2. 
20. Michael CW, Kameyama K, Kitagawa W, Azar N. Rapid on-site evaluation (ROSE) for fine needle aspiration of thyroid: benefits, challenges and innovative solutions. Gland Surg 2020;9:1708-1715. https://doi.org/10.21037/ gs-2019-catp-23.

21. Ahmadi S, Oyekunle $T$, Jiang $X$, Scheri R, Perkins J, Stang $\mathbf{M}$, et al. A direct comparison of the ATA and TI-RADS ultrasound scoring systems. Endocr Pract 2019;25:413-422. https:// doi.org/10.4158/ep-2018-0369.

22. Melany M, Chen S. Thyroid cancer: Ultrasound imaging and fine-needle aspiration biopsy. Endocrinol Metab Clin North Am 2017;46:691711. https://doi.org/10.1016/j.ecl.2017.04.011.

23. Tessler FN, Middleton WD, Grant EG, Hoang JK, Berland LL, Teefey SA, et al. ACR Thyroid Imaging, Reporting and Data System (TI-RADS): white paper of the ACR TI-RADS committee. J Am Coll Radiol 2017;14:587-595. https://doi. org/10.1016/j.jacr.2017.01.046.

24. Fiore E, Rago T, Provenzale MA, Scutari M, Ugolini C, Basolo F, et al. Lower levels of TSH are associated with a lower risk of papillary thyroid cancer in patients with thyroid nodular disease: thyroid autonomy may play a protective role. Endocr Relat Cancer 2009;16:12511260. https://doi.org/10.1677/erc-09-0036.

25. Meller J, Becker W. The continuing importance of thyroid scintigraphy in the era of high-resolution ultrasound. Eur J Nucl Med Mol Imaging 2002;29:S425-438. https://doi. org/10.1007/s00259-002-0811-8.

26. Matthew T, Olson MAZ. Thyroid fine-needle aspiration and cytological diagnosis. In: Jameson JL, De Groot L, eds. Endocrinology: Adult and pediatric. 7th ed: Elsevier; 2016. p. 1417-1422.

27. Yang W, Fananapazir G, LaRoy J, Wilson M, Campbell MJ. Can The American Thyroid Association, K-TIRADS and ACR-TIRADS ultrasound classification systems be used to predict malignancy in Bethesda category IV nodules? Endocr Pract 2020. https://doi.org/10.4158/ep-2020-0024.

28. Tan GH, Gharib H. Thyroid incidentalomas: management approaches to nonpalpable nodules discovered incidentally on thyroid imaging. Ann Intern Med 1997;126:226-231. https://doi.org/10.7326/0003-4819-126-3199702010-00009.
29. Singaporewalla RM, Hwee J, Lang TU, Desai V. Clinico-pathological correlation of thyroid nodule ultrasound and cytology using the TIRADS and Bethesda classifications. World J Surg 2017;41:1807-1811. https://doi. org/10.1007/s00268-017-3919-5.

30. Guth S, Theune U, Aberle J, Galach A, Bamberger CM. Very high prevalence of thyroid nodules detected by high frequency $(13 \mathrm{MHz})$ ultrasound examination. Eur J Clin Invest 2009;39:699-706. https://doi.org/10.1111/ j.1365-2362.2009.02162.x.

31. Pastorello RG, Destefani C, Pinto PH, Credidio CH, Reis RX, Rodrigues TA, et al. The impact of rapid on-site evaluation on thyroid fine-needle aspiration biopsy: A 2-year cancer center institutional experience. Cancer Cytopathol 2018;126:846-852. https://doi. org/10.1002/cncy.22051.

32. Haugen BR, Alexander EK, Bible KC, Doherty GM, Mandel SJ, Nikiforov YE, et al. 2015 American Thyroid Association management guidelines for adult patients with thyroid nodules and differentiated thyroid cancer: The American Thyroid Association Guidelines Task Force on thyroid nodules and differentiated thyroid cancer. Thyroid 2016;26:1-133. https:// doi.org/10.1089/thy.2015.0020.

33. Detweiler K, Elfenbein DM, Mayers D. Evaluation of thyroid nodules. Surg Clin North Am 2019;99:571-586. https://doi.org/10.1016/j. suc.2019.04.001.

34. Vitagliano G, Santoro G, Landolfi L, Cozzolino I, Peluso AL, leni A, et al. Fine-needle cytology of intraglandular parotid lymph node: A useful procedure in the management of salivary gland nodules. Diagn Cytopathol 2019;47:695700. https://doi.org/10.1002/dc.24177.

35. Horvath L, Kraft M. Evaluation of ultrasound and fine-needle aspiration in the assessment of head and neck lesions. Eur Arch Otorhinolaryngol 2019;276:2903-2911. https://doi. org/10.1007/s00405-019-05552-z.

36. Sakairi Y, Nakajima T, Yoshino I. Role of endobronchial ultrasound-guided transbronchial needle aspiration in lung cancer management. Expert Rev Respir Med 2019;13:863870. https://doi.org/10.1080/17476348.201 9.1646642 . 
37. Jain D, Allen TC, Aisner DL, Beasley MB, Cagle PT, Capelozzi VL, et al. Rapid on-site evaluation of endobronchial ultrasound-guided transbronchial needle aspirations for the diagnosis of lung cancer: A perspective from members of the Pulmonary Pathology Society. Arch Pathol Lab Med 2018;142:253-262. https://doi. org/10.5858/arpa.2017-0114-SA.

38. Ravaioli S, Bravaccini S, Tumedei MM, Pironi F, Candoli P, Puccetti M. Easily detectable cytomorphological features to evaluate during ROSE for rapid lung cancer diagnosis: from cytology to histology. Oncotarget 2017;8:11199-11205. https://doi. org/10.18632/oncotarget.13204.

39. Bilaçeroğlu S. Molecular markers in lung cancer: role of EBUS. Curr Opin Pulm Med 2017;23:247-253. https://doi.org/10.1097/ mcp.0000000000000376.

40. Itonaga $\mathbf{M}, \mathbf{A s h i d a} \mathbf{R}$, Kitano $\mathbf{M}$. Endoscopic ultrasound-guided fine-needle aspiration (EUSFNA) with image enhancement. Diagnostics 2020;10:888. https://doi.org/10.3390/diagnostics10110888.

41. Coe A, Conway J, Evans J, Goebel M, Mishra G. The yield of EUS-FNA in undiagnosed upper abdominal adenopathy is very high. J Clin Ultrasound 2013;41:210-213. https://doi. org/10.1002/jcu.22013.

42. Walia S, Aron M, Hu E, Chopra S. Utility of rapid on-site evaluation for needle core biopsies and fine-needle aspiration cytology done for diagnosis of mass lesions of the liver. J Am Soc Cytopathol 2019;8:69-77. https://doi. org/10.1016/j.jasc.2018.08.001.

43. Selhi PK, Tyagi R, Bansal P, Kaur H, Sood N. Hepatic fine-needle aspiration cytology: The role of rapid on-site evaluation in the assessment of hepatic lesions. Turk J Gastroenterol 2018;29:442-447. https://doi.org/10.5152/ tjg.2018.17466.

44. Hikichi T, Irisawa A, Bhutani MS, Takagi T, Shibukawa G, Yamamoto G, et al. Endoscopic ultrasound-guided fine-needle aspiration of solid pancreatic masses with rapid on-site cytological evaluation by endosonographers without attendance of cytopathologists. J Gastroenterol 2009;44:322-328. https://doi.org/10.1007/ s00535-009-0001-6.
45. de Moura DTH, McCarty TR, Jirapinyo $P$, Ribeiro IB, Hathorn KE, Madruga-Neto AC, et al. Evaluation of endoscopic ultrasound fineneedle aspiration versus fine-needle biopsy and impact of rapid on-site evaluation for pancreatic masses. Endosc Int Open 2020;8:e738747. https://doi.org/10.1055/a-1122-8674.

46. Shi J, Lew M, Zalupski MM, Roh MH, Kwon RS, Pang JC. Implication of suspicious cytology in endoscopic ultrasound-guided fine-needle aspiration for pancreatic cancer. J Gastrointest Canc 2015;46:54-59. https://doi.org/10.1007/ s12029-014-9681-0.

47. Ali S, Hawes RH, Kadkhodayan K, Rafiq E, Navaneethan U, Bang JY, et al. Utility of rapid onsite evaluation of touch imprint cytology from endoscopic and cholangioscopic forceps biopsy sampling (with video). Gastrointest Endosc 2019;89:340-344. https://doi.org/10.1016/j. gie.2018.08.050.

48. Lin DM, Tracht J, Rosenblum F, Kouba E, Bahl D, Patel A, et al. Rapid on-site evaluation with telecytology significantly reduced unsatisfactory rates of thyroid fine-needle aspiration. Am J Clin Pathol 2020;153:342-345. https://doi. org/10.1093/ajcp/aqz164.

49. Lin O, Rudomina D, Feratovic R, Sirintrapun SJ. Rapid on-site evaluation using telecytology: A major cancer center experience. Diagn Cytopathol 2019;47:15-19. https://doi. org/10.1002/dc.23925.

50. Gutmann EJ. Something's lost, but something's gained: On-site evaluation, telecytology, and the cytopathologist. Cancer Cytopathol 2019;127: 222-224. https://doi.org/10.1002/cncy.22111.

51. Lin O. Telecytology for rapid on-site evaluation: Current status. J Am Soc Cytopathol 2018;7:16. https://doi.org/10.1016/j.jasc.2017.10.002.

52. Ohori NP, Landau MS, Manroa P, Schoedel KE, Seethala RR. Molecular-derived estimation of risk of malignancy for indeterminate thyroid cytology diagnoses. J Am Soc Cytopathol 2020;9:213-220. https://doi. org/10.1016/j.jasc.2020.03.004.

53. Sanchez A, Bocklage T. Precision cytopathology: expanding opportunities for biomarker testing in cytopathology. J Am Soc Cytopathol 2019;8:95-115. https://doi. org/10.1016/j.jasc.2018.12.003. 